\title{
Comportement non drainé du sable d'Hostun lâche
}

\author{
Undrained behaviour of loose Hostun sand
}

\author{
J.-M. KONRAD \\ Professeur agrégé, Université Laval, Québec \\ E. FLAVIGNY \\ Maître de conférences à l'Université de Grenoble** \\ M. MEGHACHOU \\ Chercheur $3^{\circ}$ cycle* *
}

Rev. Franç. Géotech. n 54 , pp. 53-63 (janvier 1991)

\section{Résumé}

Les résultats d'essais consolidés non drainés sur des échantillons reconstitués de sable d'Hostun sont analysés pour mettre en évidence les conditions qui conduisent au radoucissement du sable sous charge monotone. Le programme d'essais confirme l'existence d'une limite supérieure et d'une limite inférieure pour la ligne «F " définie par CASTRO pour caractériser l'état d'écoulement permanent. Ces deux limites sont appelées ligne UF et ligne LF, respectivement. La résistance minimum non drainée du sable d'Hostun correspond à la ligne LF et dépend de l'indice des vides et de la pression de consolidation, c'est-à-dire d'un paramètre d'état initial. Ces résultats confirment ceux déjà obtenus par KONRAD (1990).

\section{Abstract}

Results of CIU tests on reconstituted samples of Hostun sand are presented to establish the conditions to induce strain-softening during monotonic loading. The test program confirmed the existence of an upper and lower limit of the $F$ line defined by CASTRO to characterize the conditions of steady state deformation in loose sands. These limits were refferred to as the UF line and LF line, respectively. The minimum undrained shear strength of Hostun sand corresponds to the LF line and is dependent upon both the void ratio and the consolidation pressure; i.e. upon the initial state parameter. Similar results were obtained on different sands by KONRAD (1990).

* 513378 Québec Canada G 1K7P4.

* Domaine universitaire, BP n $53 \mathrm{X}, 38041$ Grenoble Cedex. 


\section{INTRODUCTION}

Le concept d'état critique pour les matériaux sableux a été introduit dès 1936 par CASAGRANDE (1936) et correspond à l'indice des vides d'un échantillon de sable cisaillé en conditions drainées lorsqu'il est soumis à de très grandes déformations. Un échantillon de sable dense dilatera lors du cisaillement et un échantillon de sable lâche contractera. L'observation de l'indice des vides critique sur les matériaux denses peut être masquée par l'apparition prématurée de surface de cisaillement que l'utilisation d'échantillons antifrettés retarde considérablement (cf. COLLIAT. DESRUES, FLAVIGNY, 1986). Cette propriété dépend aussi du niveau de la contrainte isotrope de consolidation (ou du niveau de la contrainte normale). Le diagramme critique a été défini par BIAREZ et GRÉSILLON (1972) et FORAY et PUECH (1976) comme la relation entre l'indice des vides initial et la pression de consolidation ou la contrainte movenne en fin d'essai pour des essais drainés sous contrainte latérale constante. FLAVIGNY, FORAY et DARVE (1979) comparent les diagrammes critiques tels qu'on peut les déduire d'essais drainés et d'essais non drainés : en effet dans un essai non drainé, par conséquent à indice des vides constant, on observe des variations de pression interstitielle telles que la pression moyenne en fin d'essai soit en accord avec le diagramme critique. Ces travaux rejoignent ceux de CASTRO (1969). Plus récemment, à la suite des ruptures d'îles artificielles initiées par une liquéfaction statique de dépôts sableux lâches, BEEN et JEFFERIES (1988) ont défini un paramètre d'état tandis que SLADEN, D'HOLLANDER et KRAHN (1985) introduisaient le concept de surface d'effondrement. Ces travaux, uniquement relatifs à des matériaux sableux lâches et très lâches sont brièvement rappelés cidessous, à partir du concept « d'état permanent de déformation " qu'il convient de replacer dans le cadre de la théorie de la plasticité parfaite, correspondant à l'écoulement plastique d'un matériau, sous état de contrainte constant et à volume constant. On trouvera aussi dans l'état de l'art de SCHLOSSER, CORTÉ et DORMIEUX (1987) l'application de ces concepts à la liquéfaction statique des sables.

\section{CONCEPT D'ÉTAT PERMANENT DE DÉFORMATION}

En 1969. CASTRO présente les résultats d'une série d'essais triaxiaux non drainés sur des échantillons de sable consolidés de manières isotrope et anisotrope à différents indices des vides et cisaillés à contrainte contrôlée. Il met en évidence des réductions importantes de la résistance au cisaillement non drainée pour les sables lâches. La figure 1a présente dans les axes $\left(\sigma_{1}-\sigma_{3}, \epsilon_{1}\right)$ des résultats typiques d'essais non drainés consolidés à $400 \mathrm{kPa}$. L'essai (a), avec la plus faible densité relative, présente un pic de résistance pour une déformation axiale d'environ $1 \%$, suivi d'une chute importante et établissement d'une résistance au cisaillement constante pour des déformations supérieures à $5 \%$. La pression interstitielle passe d'abord par une phase de génération importante à taux élevé, puis se stabilise à une valeur constante
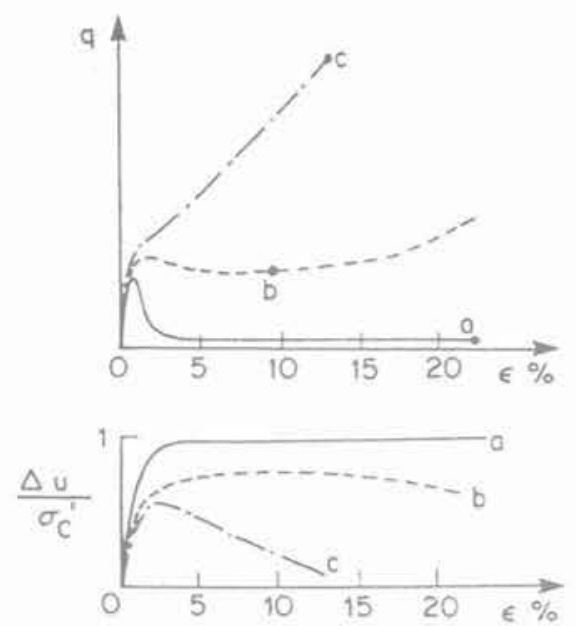

Fig. 1a. - Courbes schématiques d'essais triaxiaux non drainés sur sable (d'après CASTRO, 1969).

Fig. 1a. - Tvoical curves for undrained triaxial tests on sand lafter CASTRO, 1969).

pour des déformations supérieures à $3 \%$. Donc, un état de plasticité parfaite est atteint pour lequel l'échantillon se déforme à volume constant, résistance au cisaillement non drainée constante et pression interstitielle constante, c'est-à-dire état de contraintes effectives constantes. CASTRO a établi que la résistance non drainée à l'état de plasticité parfaite ne dépendait que de lindice des vides initial du sable. Il a aussi suggéré que les conditions de contraintes à l'état de plasticité parfaite pouvaient être définies par la valeur de $\sigma_{3}^{\prime}$ en fin d'essai. Par conséquent, le lieu de l'état de plasticité parfaite dans un plan e-Log $\sigma_{3}^{\prime}$ était une courbe unique appelée courbe $\mathrm{F}$ tel qu'illustré à la figure 1b. CASAGRANDE (1936) avait postulé que le comportement non drainé à l'état de plasticité parfaite (état critique à l'époque) correspondait au développement d'une « structure d'écoulement » d'où l'utilisation de la lettre F (flow) dans les diagrammes d'état de plasticité parfaite.

L'essai (c), avec la plus forte densité relative, présente une résistance au cisaillement croissant régulièrement en fonction des déformations, tandis que la pression

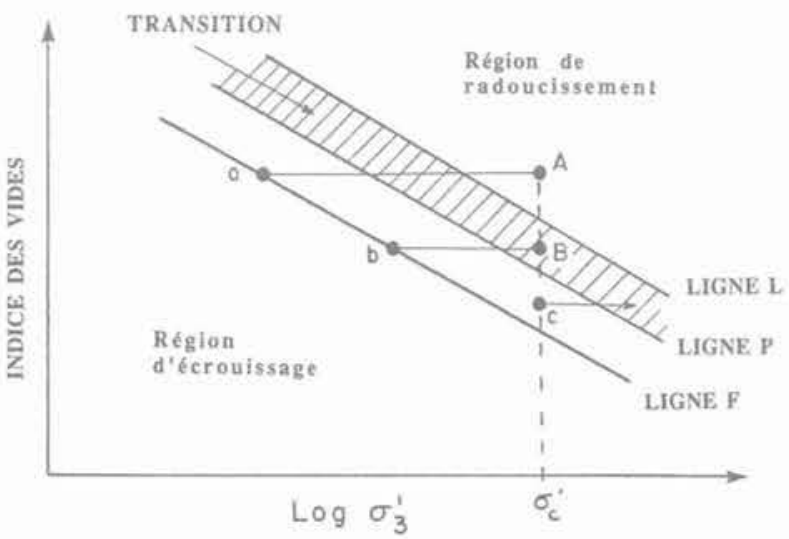

Fig. 1b. - Interprétation dans un diagramme d'état: indice des vides et pression de consolidation.

Fig. 1b. - Interpretation in a state diagram: voids ratio vs consolidation pressure lafter CASTRO, 1969). 
interstitielle passe par un maximum avant de décroître, pouvant même devenir inférieure à la pression atmosphérique.

Pour l'essai (b), de densité intermédiaire par rapport aux précédentes, on observe un pic de résistance suivi d'une faible chute et un palier où la résistance au cisaillement est constante jusqu'à des déformations axiales d'environ $10 \%$. Cependant, cette dernière augmente légèrement lorsque les déformations continuent d'évoluer.

CASTRO (1969) définit dans le plan e-Log $\sigma_{3}^{\prime}$ une région de transition située entre les courbes $\mathrm{P}$ et $\mathrm{L}$ (fig. 1b) qui sont parallèles à la courbe $F$ représentant le lieu de l'état de plasticité parfaite. Lorsque les échantillons de sable sont au-dessus de la courbe L, ils seront caractérisés par des courbes de résistance non drainée présentant un radoucissement important jusqu'à l'état permanent défini par la courbe F. Si les échantillons sont en-dessous de la courbe $\mathrm{P}$, is seront alors dilatants en essais drainés et présenteront des courbes de résistance au cisaillement non drainée avec écrouissage (essais de type (c)). Les échantillons dans la zone de transition développeront des courbes du type (b). D'après CASTRO, la valeur de la résistance au cisaillement non drainée correspondant au palier est toujours définie par la courbe F.

Dans ces conditions, pour un indice des vides donné, le niveau de contrainte isotrope initial n'a pas d'influence sur la contrainte effective principale mineure à l'état permanent dans un essai non drainé pour autant que le niveau de contrainte isotrope soit supérieur à une certaine valeur définie par la courbe $\mathrm{P}$ dans le diagramme e- $\log \sigma_{3}^{\prime}$ (fig. Ib).

Depuis quelques années, les conditions à l'état permanent sont exprimées en fonction de la contrainte effective moyenne, $\mathrm{p}_{\mathrm{s}}^{\prime}$. l'indice $\mathrm{s}$ désignant le "steady state of flow ", ce qui a l'avantage d'inclure la valeur du déviateur de contrainte ainsi que celle de la pression interstitielle à l'état de plasticité parfaite. Plusieurs études (BEEN et JEFFERIES, 1895 ; KRAMER et SEED, 1988 ; et CANOU, 1989) ont également confirmé que, pour un indice de vide donné, le niveau de contrainte isotrope initial n'avait pas d'influence sur la contrainte effective moyenne à l'état de plasticité parfaite, référée par p's. Cependant, peu d'essais ont été effectués dans la zone de transition entre les courbes $\mathrm{P}$ et L. Dans cette zone, les résultats de CASTRO montrent que les essais ne présentent pas un comportement en plasticité parfaite, mais que la contrainte minimum (point $b$ sur la figure 1) se situe sur la courbe d'état de plasticité parfaite.

\section{CONCEPT MODIFIÉ DU COMPORTEMENT NON DRAINÉ DES SABLES LÂCHES}

KONRAD (1990) a étudié l'influence du niveau de contrainte isotrope initial sur les caractéristiques de deux sables avec une attention particulière dans la zone de transition. Ces études ont permis de démontrer que, contrairement aux études antérieures, pour un indice de vide donné, le niveau de contrainte isotrope initial contrôlait les caractéristiques de déformation d'un sable lâche. De plus, les contraintes effectives moyennes à l'état de plasticité parfaite dépendaient également du niveau de contrainte isotrope initial. En d'autres termes, la courbe $F$ dans un diagramme e-Log p' n'est pas unique pour un sable donné. Les essais ont démontré l'existence d'une limite supérieure et inférieure de la résistance au cisaillement non drainée à l'état de plasticité parfaite (fig. 2) : la courbe $F$ de la figure 1 se divise en deux

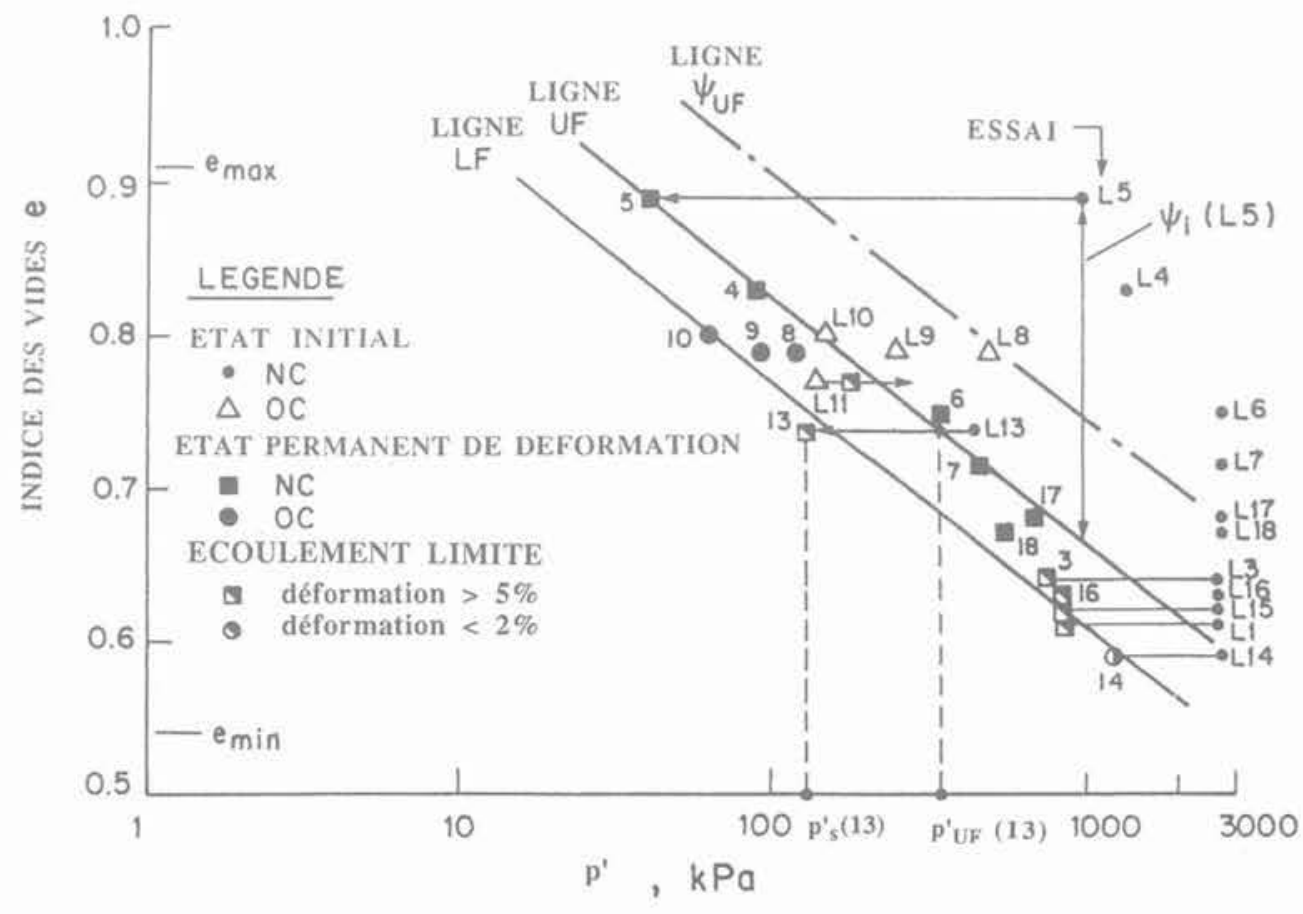

Fig. 2. - Résultats d'essais triaxiaux sur sable de dune (d'après KONRAD, 1990).

Fig. 2. - Results of triaxial test on a dune sand (after KONRAD, 1990). 
droites notées UF et LF. La limite supérieure de la résistance au cisaillement non drainée à l'état de plasticité parfaite sera désignée par la courbe UF et la limite inférieure par la courbe LF (fig. 2).

L'existence de plusieurs courbes caractérisant l'état de plasticité parfaite pour un même sol contraint alors à redéfinir le paramètre d'état $\Psi$ introduit dans la littérature par BEEN et JEFFERIE (1985). On propose alors d'utiliser la courbe UF comme droite de référence. Par conséquent, le paramètre d'état en fin de consolidation isotrope, $\Psi i$, est donné par :

$$
\Psi \mathrm{i}=e_{\mathrm{c}}-e_{\mathrm{UF}}
$$

où $e_{\mathrm{c}}$ est l'indice des vides à un niveau de contrainte isotrope de consolidation $\mathrm{p}_{\mathrm{c}}$ et et $e_{\mathrm{UF}}$ est l'indice des vides sur la courbe UF sous la même contrainte moyenne $\mathrm{p}_{\mathrm{c}}$, l'indice c désignant l'état consolidé.

Les études menées par KONRAD (1990) ont permis d'établir que des échantillons de sable présentant des paramètres d'état supérieurs à une certaine valeur $\Psi$ (UF) étaient caractérisés par une courbe de résistance au cisaillement telle, que les courbes (A) et (B) sur la figure 3 , présentant un pic de résistance pour une déformation assez faible $\left(\epsilon_{1}=1\right.$ à $\left.2 \%\right)$ suivi d'un radoucissement important se stabilisant à une valeur constante (point a) au-delà d'une certaine

(o)

(b)
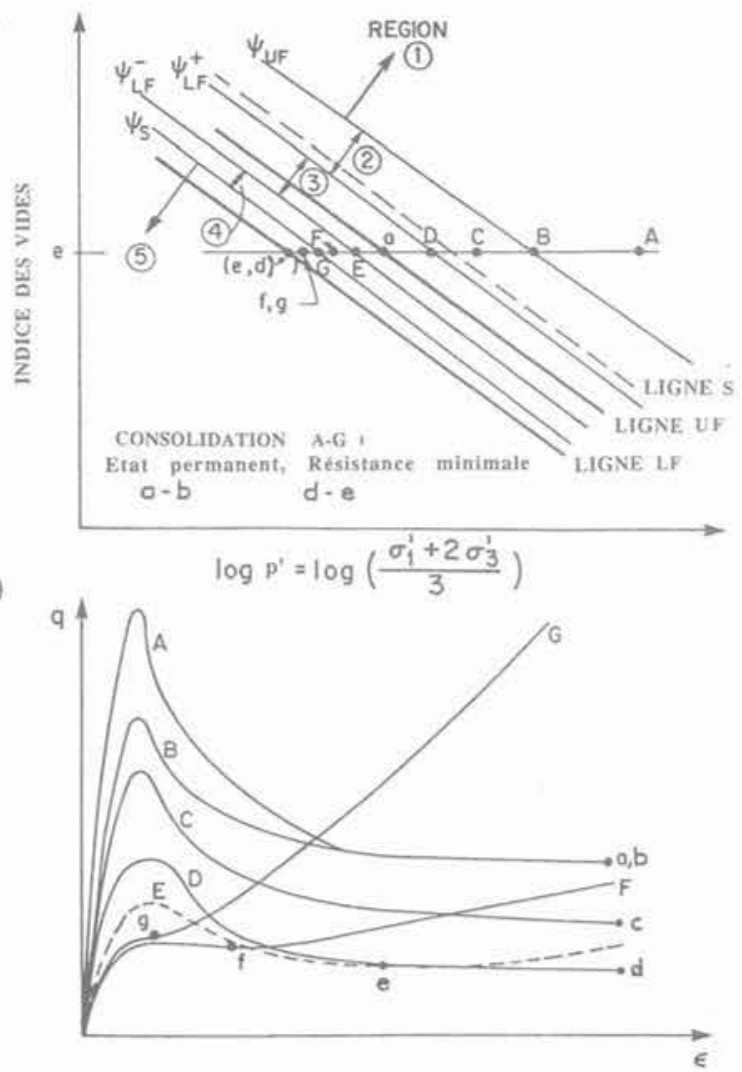

Fig. 3. - Comportements typiques de sables sous conditions non drainées : mise en évidence des lignes UF et LF (d'après KONRAD, 1990).

Fig. 3. - Typical behavior of sands under undrained triaxial loading : definition of the lines UF and LF (after KONRAD, 1990). déformation $\left(\epsilon_{1}>5\right.$ à $\left.10 \%\right)$. Dans un plan e-Log p', le lieu de ces états de plasticité parfaite correspond à la courbe UF. Lorsque les échantillons ont des paramètres d'état inférieurs à $\Psi(\mathrm{UF})$, les courbes de résistance au cisaillement non drainée n'aboutissent plus au même état de plasticité parfaite. Si le point représentatif de l'état physique du sable se trouve dans la région 3 (fig. 3), la résistance au cisaillement non drainée à l'état de plasticité parfaite sera donnée par la courbe LF, donc par le point (d) pour un indice des vides donné (fig. 3). Lorsque le point représentatif de l'état physique se situe dans la zone 2, la résistance au cisaillement correspondant à l'état de plasticité parfaite adoptera une valeur intermédiaire entre la résistance définie par la courbe UF (point a) et celle définie par la courbe LF (point d), qui dépendra de la valeur de $\Psi \mathrm{i}$.

On notera que pour une valeur de $\Psi$ i proche de la limite inférieure de la région 3 , l'échantillon de sable sera caractérisé par une courbe de résistance présentant un pic pour une déformation axiale de l'ordre de $1 \%$ suivi d'un faible radoucissement pour atteindre un palier jusqu'à des déformations de l'ordre de 10 à $15 \%$. Au-delà de ces déformations, les pressions interstitielles diminuent légèrement et la résistance au cisaillement augmente. Ce phénomène a été observé par CASTRO qui l'a identifié comme un « écoulement limité » ou un état quasi-plastique.

Finalement, tout échantillon de sable dans la région 5 sera caractérisé par des courbes de résistance non drainée du type (G) (fig. 3) présentant une augmentation monotone de la résistance au cisaillement en fonction de la déformation. Cette résistance tend vers l'état critique qui n'est pas forcément identique à l'état de plasticité parfaite obtenu avec des essais non drainés tel que suggéré par CASTRO (1969) et ALARCON-GUZMAN et al, (1989). Ce point sera traité dans une autre communication.

KONRAD (1990) a également démontré que la position relative des régions 1 à 5 dans le plan e-Log p' dépend du type de sable.

\section{PROGRAMME EXPÉRIMENTAL}

Dans le but d'établir les valeurs $\Psi(\mathrm{UF}), \Psi(\mathrm{LF}+)$ et $\Psi(\mathrm{LF}-)$ pour le sable d'Hostun RF qui est un sable de référence utilisé depuis plusieurs années par l'IMG, le programme expérimental comprend deux séries d'essais dans la cellule triaxiale. La première était caractérisée par des essais non drainés à différents indices des vides et à des niveaux de contrainte isotrope suffisamment élevés pour déterminer la courbe UF. La deuxième série comprenait des essais sur des échantillons à indice des vides plus ou moins constant soumis à différents niveaux de contrainte isotrope afin de déterminer les positions relatives des régions 1 à 5 pour le sable d'Hostun.

Les conditions expérimentales sont présentées dans le tableau 1. 
Tableau 1.

\begin{tabular}{|c|c|c|c|c|c|c|c|}
\hline$N^{0}$ d'essai & $e_{0}$ & $e_{\text {cons }}$ & $\begin{array}{c}\sigma_{\mathrm{c}} \\
\mathrm{kPa}\end{array}$ & $\frac{\Delta \mathrm{u}}{\sigma_{\mathrm{c}}}$ & $\begin{array}{l}\mathrm{p}^{\prime} \mathrm{s} \\
\mathrm{kPa}\end{array}$ & $\begin{array}{l}\mathrm{q}^{\prime} \mathrm{s} \\
\mathrm{kPa}\end{array}$ & Remarques \\
\hline 6 & 1,04 & 1,009 & 288 & 0,99 & 7,12 & 10,5 & \multirow{3}{*}{$\begin{array}{l}\sigma_{\mathrm{c}} \text { constant } \\
\text { Figures } 4 \mathrm{a} \text {, b et figure } 5\end{array}$} \\
\hline 8 & 1,010 & 0,970 & 279 & 0,96 & 22 & 33,5 & \\
\hline 36 & 0,926 & 0,914 & 269 & 0,93 & 30,8 & 38 & \\
\hline 24 & 0,926 & 0,917 & 175 & 0,91 & 23,5 & 22,8 & \multirow{5}{*}{$e_{0}=$ constant } \\
\hline 25 & 0,980 & 0,922 & 772 & 0,95 & 57,0 & 61,0 & \\
\hline 34 & 0,925 & 0,918 & 137 & 0,92 & 20,70 & 30,8 & \\
\hline 35 & 0,931 & 0,928 & 28 & 0,73 & 13,5 & 18,0 & \\
\hline 36 & 0,926 & 0,914 & 269 & 0,93 & 30,8 & 38,8 & \\
\hline 37 & 0,948 & 0,928 & 397 & 0,93 & 49,0 & 68,3 & Figures $7 a, b$ et 8 \\
\hline 17 & 0,876 & 0,834 & 750 & 0,8 & 248,5 & 294,8 & \multirow{2}{*}{$\begin{array}{c}\sigma_{c} \text { constant } \\
\sigma_{c} \text { grand }\end{array}$} \\
\hline 25 & 0,980 & 0,922 & 772 & 0,95 & 57 & 61 & \\
\hline
\end{tabular}

Le sable d'Hostun (RF) est un sable de carrière, quartzeux à grains sub-anguleux, de granulomètrie uniforme. Les principales caractéristiques physiques du sable d'Hostun RF sont détaillées dans FLAVIGNY, DESRUES, PALAYER (1990).

\section{PROCÉDURE EXPÉRIMENTALE}

\subsection{Préparation des échantillons}

La difficulté majeure des essais réalisés antérieurement (DJEDID, 1986 ; BOUSQUET, 1988) à l'IMG dans le cadre de l'étude du comportement non drainé du sable d'Hostun consistait à obtenir des structures lâches contractantes pendant le cisaillement. La préparation des échantillons par la méthode de déversement sec à hauteur de chute nulle ne donnait pas des structures suffisamment lâches après saturation et consolidation isotrope. Si l'on utilise un sable légèrement humide (teneur en eau de 2 à $4 \%$ ), on peut atteindre un indice des vides nettement supérieur à $e_{\max }$ obtenu avec le sable sec selon les normes en vigueur. On peut attribuer cet état très lâche à la cohésion apparente développée par les succions capillaires.

Les échantillons de sable ont été par conséquent préparés avec un sable à une teneur en eau de $2 \%$ et un moule cylindrique de $100 \mathrm{~mm}$ de diamètre et de hauteur, donnant un élancement égal à 1. On applique le vide entre la paroi du moule et la membrane de latex pour plaquer cette dernière à la paroi, et on dépose le matériau à l'aide d'une petite cuillère en couches successives. Le remplissage du moule se fajsait en 5 couches dont la masse de chacune est déterminée au préalable. Chaque couche est compactée jusqu'à ce que son épaisseur soit égale à une valeur conforme avec la densité visée.

\subsection{Saturation et consolidation isotrope}

La saturation de l'éprouvette de sable comporte deux phases. La première consiste à remplacer l'air contenu dans les vides par du dioxyde de carbone que l'on fait circuler à travers l'échantillon sous faible pression (environ 15 à $20 \mathrm{kPa}$ ) pendant 20 minutes. Ensuite, on fait circuler de l'eau désaérée pendant au moins 20 minutes. On notera que durant cette phase il est quasiment impossible de déterminer les changements de volume de l'échantillon qui seront d'autant plus importants que l'indice des vides sera plus grand.

La deuxième phase consiste à appliquer une contrepression de $450 \mathrm{kPa}$ qui dissoudra le dioxyde de carbone et assurera une saturation adéquate puisque le coefficient de Skempton B était supérieur à $97 \%$ dans tous nos essais. Le niveau de contrainte isotrope désiré est obtenu en ajustant la pression cellulaire et en permettant le drainage aux extrémités de l'échantillon. Durant la consolidation, les changements de volume sont enregistrés automatiquement et les changements d'indice des vides sont calculés aisément. Avant de commencer un essai de compression, il faut toutefois s'assurer que la consolidation soit bien complète.

\subsection{Essai triaxial de compression et pénétration de membrane}

Tous les essais de compression ont été effectués dans une cellule triaxiale en condition non drainée. La charge axiale est appliquée par l'intermédiaire d'une tête rotulée et la vitesse de déplacement du plateau de la presse est égale à $1 \mathrm{~mm} / \mathrm{min}$ (soit $1 \% / \mathrm{min}$ pour l'échantillon d'élancement 1). Un sustème d'antifrettage composé de bases élargies métalliques (d'un diamètre de $120 \mathrm{~mm}$ ), polies et lubrifiées par une fine 
couche de graisse au silicone et d'une rondelle de latex a été utilisé pour tous les essais décrits dans cet article. COLLIAT et al. (1986) donnent des détails complets sur le système d'antifrettage. Ce système permet d'obtenir des déformations homogènes jusqu'à de très grandes déformations axiales.

Les pressions interstitielles générées lors du cisaillement en conditions non drainées sont importantes dans le cas de sables lâches et peuvent réduire la pression de confinement exercée sur la membrane. Ceci peut entraîner une variation de volume et induire une sousestimation des pressions interstitielles. Pour le sable d'Hostun RF, l'effet de pénétration de membrane est négligeable puisque le changement de volume pour un changement de pression de confinement de 500 $\mathrm{kPa}$ est inférieur à $1 \%$ d'après la relation compilée par SLADEN et al., (1986).

\section{RÉSULTATS DES ESSAIS NON DRAINÉS}

\subsection{Influence de la densité}

La figure $4 \mathrm{a}$ montre des courbes de résistance obtenues à l'appareil triaxial à déformation contrôlée pour des essais à différentes densités mais au même niveau de contrainte isotrope d'environ $280 \mathrm{kPa}$. Les cour-
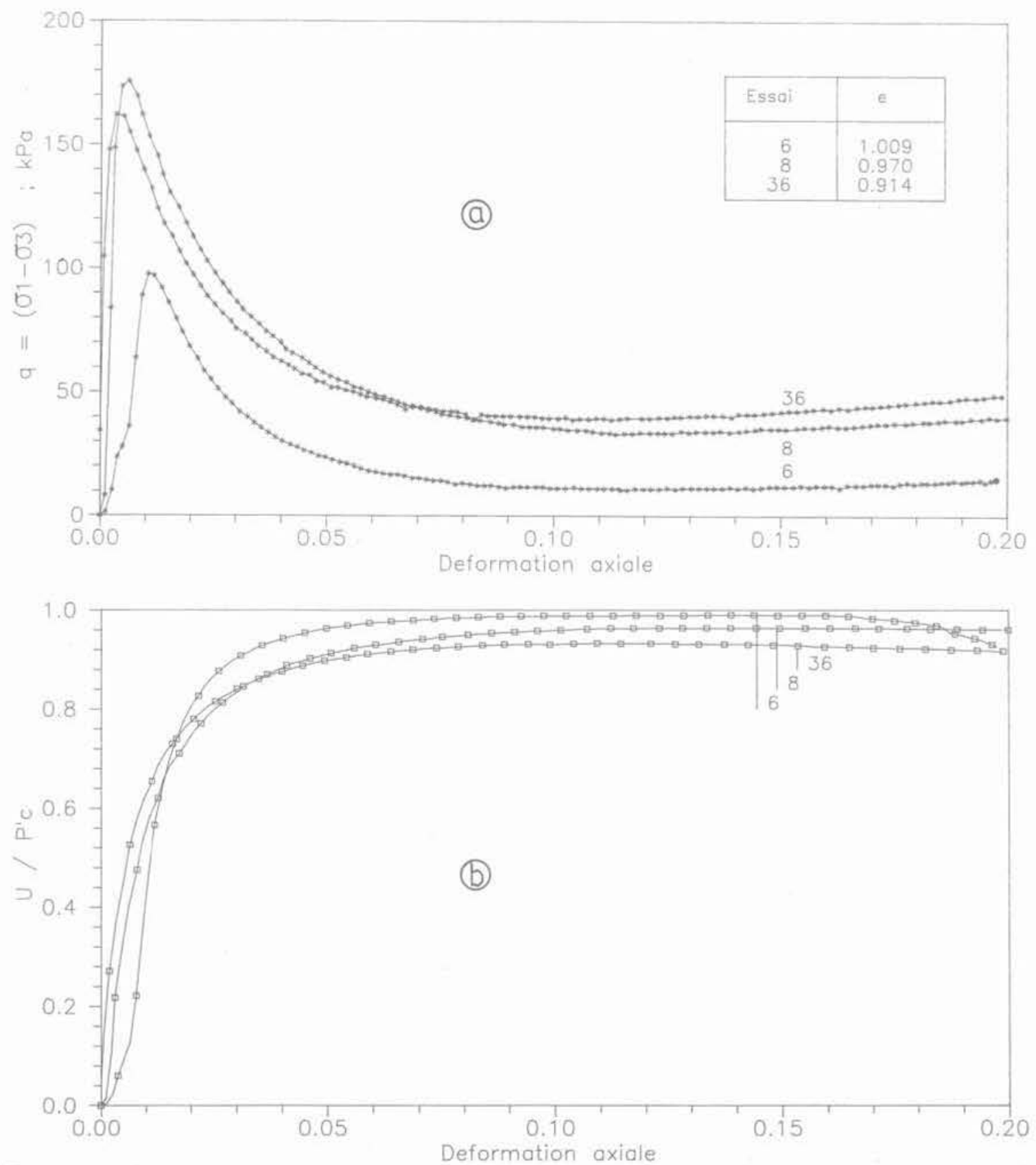

Fig. $4 a$. et $4 b$. - Courbes effort-déformation et pression interstitielle-déformation pour les essais consolidés à $280 \mathrm{kPa}$. Fig. $4 a$ and $4 \mathrm{~b}$. - Stress - strain and pore pressure - strain curves for tests consolidated under $280 \mathrm{kPa}$. 
bes sont caractérisées par un pic du déviateur de contrainte pour une déformation axiale de l'ordre de 1 à $2 \%$ suivi d'une réduction du déviateur jusqu'à l'obtention de l'état de plasticité parfaite pour lequel l'échantillon se déforme à déviateur constant. En général, l'état de plasticité parfaite est atteint pour des déformations axiales supérieures à $10 \%$. La figure $4 \mathrm{~b}$ montre l'évolution des pressions interstitielles normalisées par rapport au niveau de contrainte isotrope initial en fonction de la déformation axiale. Les surpressions atteignent une valeur stable pour des déformations supérieures à $6 \%$. On remarquera que le rapport $\Delta \mathrm{u} / \sigma^{\prime} 3$ décroît de 0,992 à 0,937 lorsque l'indice des vides diminue de 1,01 à 0,914 .

La densité du sable influence également la résistance au cisaillement non drainée à l'état de plasticité parfaite. En effet, cette dernière augmente lorsque la densité de l'échantillon augmente, c'est-à-dire lorsque son indice des vides diminue.

Les chemins de contrainte dans le plan p'-q sont illustrés sur la figure 5 et mettent en évidence la mobilisation du frottement au pic du déviateur ainsi qu'à l'état permanent. On constate que le frottement mobilisé au pic du déviateur est beaucoup plus faible que l'angle de frottement interne du sable vu que la pente de la droite passant entre l'origine et le pic du déviateur n'est que 0,5 pour l'essai 6 et 0,68 pour les deux autres. Donc, il apparaît que le frottement mobilisé au pic du déviateur soit également fonction de lindice des vides pour un niveau de contrainte isotrope donné. En revanche, le frottement mobilisé à l'état permanent est dans tous les cas voisin de l'angle de frottement interne du sable qui est environ $30^{\circ}$.

Des résultats identiques sont présentés à la figure 6 pour les essais 25 et 17 réalisés à différents indices des vides et au même niveau de contrainte, mais supérieur au précédent.
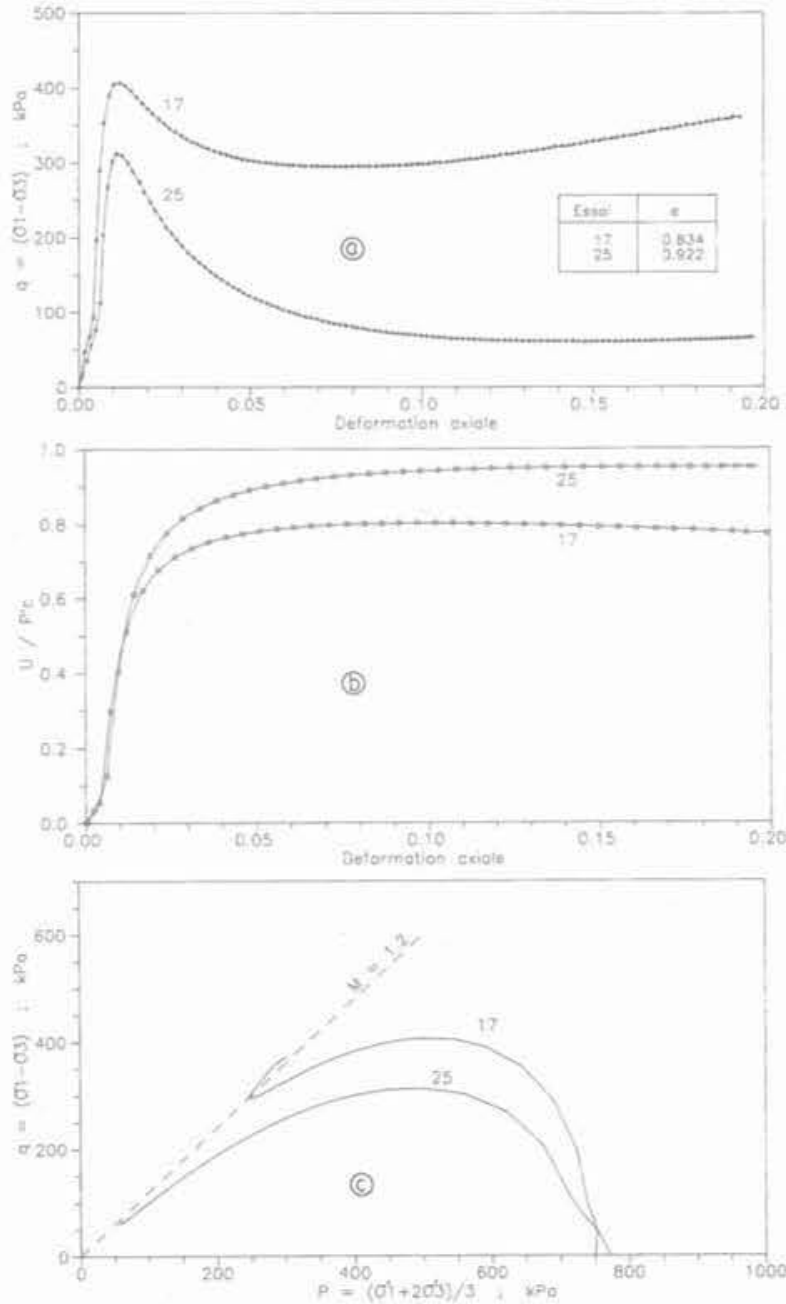

Fig. 6a., 6b. et 6c. - Courbes effort déformation et pression interstitielle déformation pour les essais consolidés à $750 \mathrm{kPa}$.

Fig. $6 a, 6 b$, and $6 c$. - Stress - Strain and pore pressure. Strain curves for tests consolidated under $750 \mathrm{kPa}$

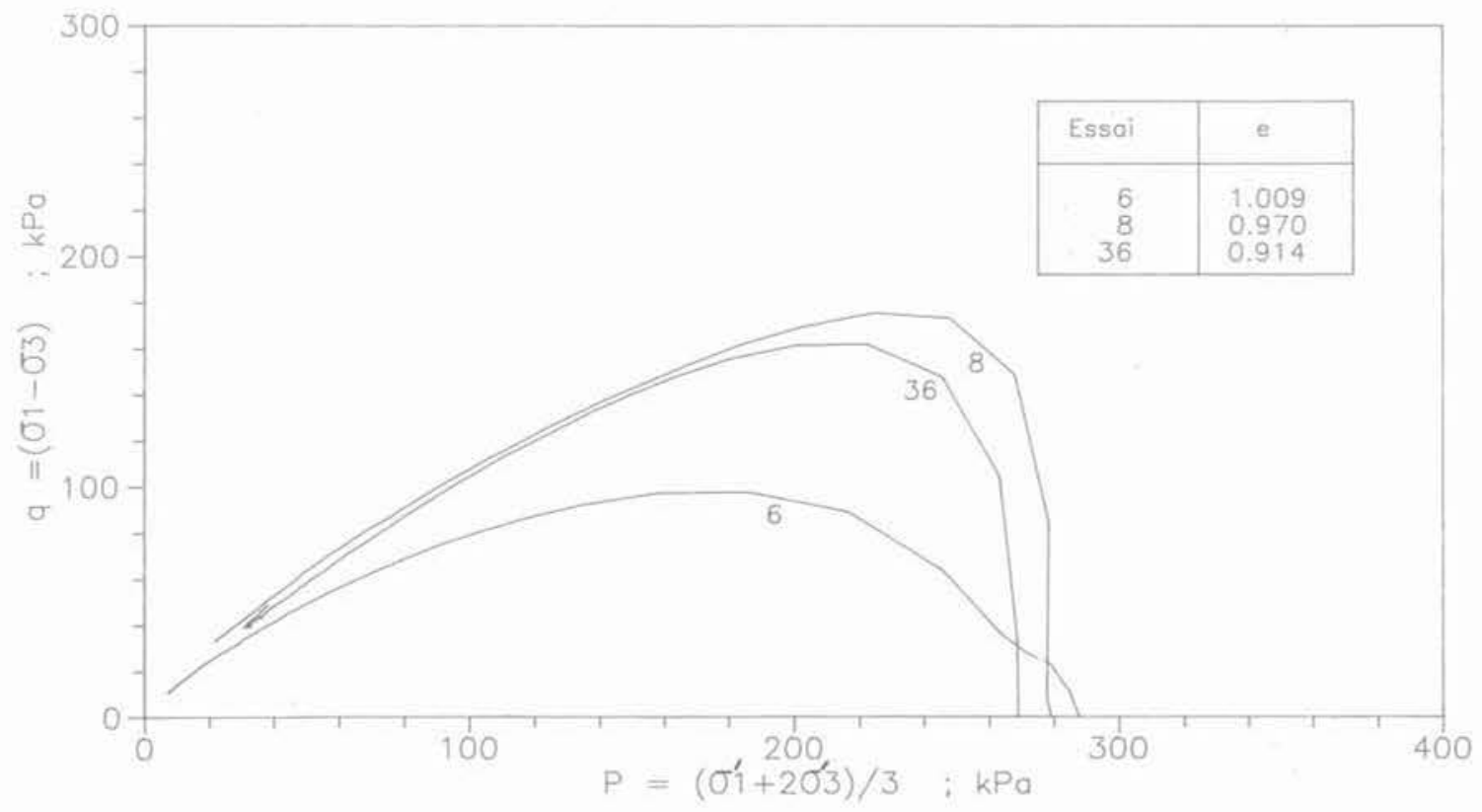

Fig. 5. - Chemins de contraintes pour les essais consolidés à $280 \mathrm{kPa}$.

Fig. 5. - Stress paths for tests consolidated under $280 \mathrm{kPa}$. 


\subsection{Influence du niveau de contrainte isotrope}

Les essais $24,25,34,35,36$ et 37 ont été réalisés sur des éprouvettes de même indice des vides (e = $0,92+/-0,007)$ et consolidés à différents niveaux de contrainte isotrope soit de 30 à $772 \mathrm{kPa}$. Les résultats de cette série d'essai sont présentés sur les figures 7 et 8 .

Le comportement non drainé de ces échantillons au même indice des vides est fonction du niveau de la contrainte isotrope après consolidation. En effet, l'essai 25 , qui était soumis à la pression de confinement la plus élevée, se caractérise par l'obtention d'un pic de déviateur pour une déformation axiale d'environ $1,5 \%$ après lequel le déviateur décroît jusqu'à l'obtention de l'état de plasticité parfaite pour une déformation de l'ordre de $10 \%$ (fig. 8a). Le déviateur à l'état permanent est égal à $60 \mathrm{kPa}$ et la pression interstitielle atteint un palier égal à $95 \%$ de la pression de confinement (fig. $8 \mathrm{~b}$ )

L'essai 35, qui a été soumis à la contrainte isotrope la plus faible, est caractérisé par un pic du déviateur très faible à une déformation axiale de l'ordre de $0,8 \%$ et d'une légère diminution de ce dernier jusqu'à l'obtention d'une valeur constante à l'état de
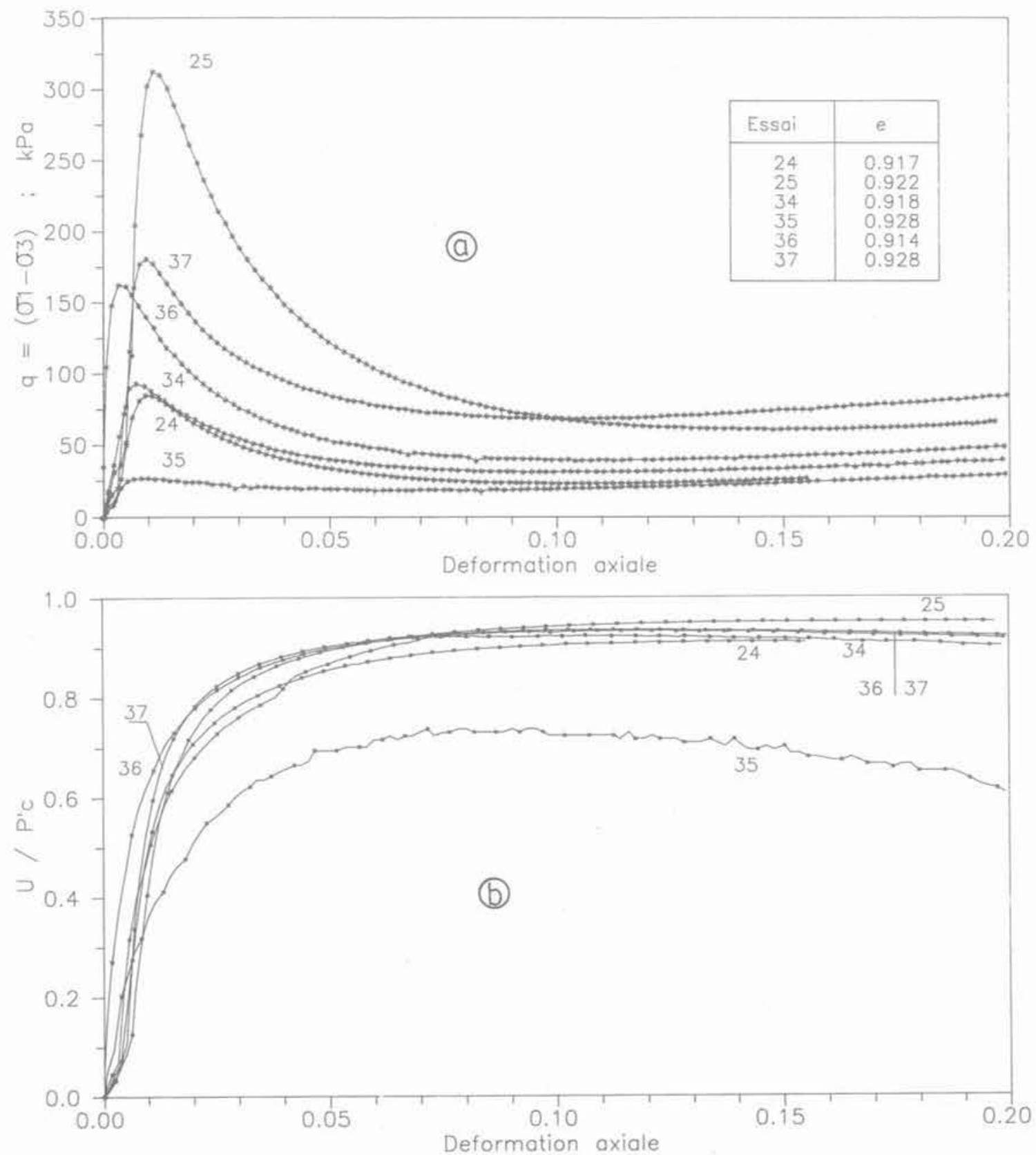

Fig. $7 a$. et $7 b$. - Courbes effort déformation et pression interstitielle déformation pour les essais consolidés à un indice des vides voisin de 0,92 sous différentes pressions de consolidation.

Fig. $7 a$. and $7 b$. - Stress - Strain and pore pressure - Strain curves for tests consolidated at a voids ratio around 0.92 under different consolidation pressures. 
plasticité parfaite égale à $20 \mathrm{kPa}$, soit le tiers du déviateur correspondant à l'état de plasticité parfaite de l'essai 25 (fig. 8a). En outre, les surpressions générées lors de l'essai 35 n'atteignent que $74 \%$ de la contrainte isotrope de confinement. Le même phénomène a déjà été observé par KONRAD (1990) sur du sable de dune très angulaire.

Les chemins de contrainte dans le plan p'-q sont illustrés sur la figure 8 . On constate que le frottement mobilisé au pic du déviateur n'est pas le même pour tous les essais de la série 2. En effet, la pente de la droite passant par les points représentatifs du pic varie entre 0,60 et 0,88 . Donc, il apparait que le frottement mobilisé au pic du déviateur soit également fonction du niveau de contrainte isotrope pour un indice des vides donné. En revanche, le frottement mobilisé à l'état permanent est dans tous les cas voisin de l'angle de frottement interne du sable qui est environ $30^{\circ}$.

\section{COURBES UF ET LF POUR LE SABLE D'HOSTUN RF}

D'après les travaux de KONRAD (1990), les échantillons dans la région 1 définissent la courbe UF correspondant à l'état de plasticité parfaite. La région 1 regroupe surtout des essais avec des niveaux de contrainte isotrope et des indices des vides relativement élevés. La courbe UF sera donc définie par les essais $5,6,8,25$ et 37 . La figure 9 montre les contraintes effectives moyennes en fonction de l'indice des vides pour ces essais. On remarquera que la courbe UF a été déterminée à l'aide des essais 8,25 et 37 pour lesquels il y a moins de risques d'effondrement durant la phase de saturation. Tel que mentionné antérieurement, lorsque les densités sont trop faibles, des changements de volume, qui ne sont pas mesurés, peuvent se produire durant la phase de saturation et par conséquent les points représentatifs des essais 5 et 6 sont probablement situés trop hauts dans le diagramme e-Log p' à la figure 9.

La courbe LF est obtenue à partir d'essais consolidés dans la région 3 (voir fig. 3). En fait c'est la limite inférieure des points représentatifs de l'état permanent. Par conséquent, la courbe LF est définie par les essais 35,15 et 17 (fig. 10). Cette courbe est parallèle à la courbe UF, ce qui confirme à la fois l'hypothèse quant à l'indice des vides des échantillons 5 et 6 ainsí que les résultats plus généraux proposés par KONRAD (1990).

La figure 9 met aussi en évidence le fait que la contrainte effective moyenne à l'état permanent des échantillons consolidés dans une région de transition située entre les régions 1 et 3 , soit les essais 20, 24, 34 et 36 . est comprise entre les valeurs correspondantes sur les courbes UF et LF pour le même indice des vides.

\section{CARACTÉRISTIQUES DE L'ÉTAT DE PLASTICITÉ PARFAITE}

Les conditions $\left(e, p_{c}^{\prime}\right)$ contrôlant les caractéristiques du sable d'Hostun à l'état de plasticité parfaite peuvent être exprimées en fonction du paramètre d'état en utilisant la définition donnée par l'équation 1. La figure 10 montre le rapport $\mathrm{p}_{5} / \mathrm{p}_{\mathrm{s}}^{\prime}$ Uf en fonction du paramètre d'état $\Psi \mathrm{i}$ pour les essais de la série 1 et 2. La valeur de p'suf correspond à la valeur de la contrainte effective moyenne sur la courbe UF pour l'indice des vides de l'essai en question alors que p', est la contrainte effective moyenne mesurée à l'état

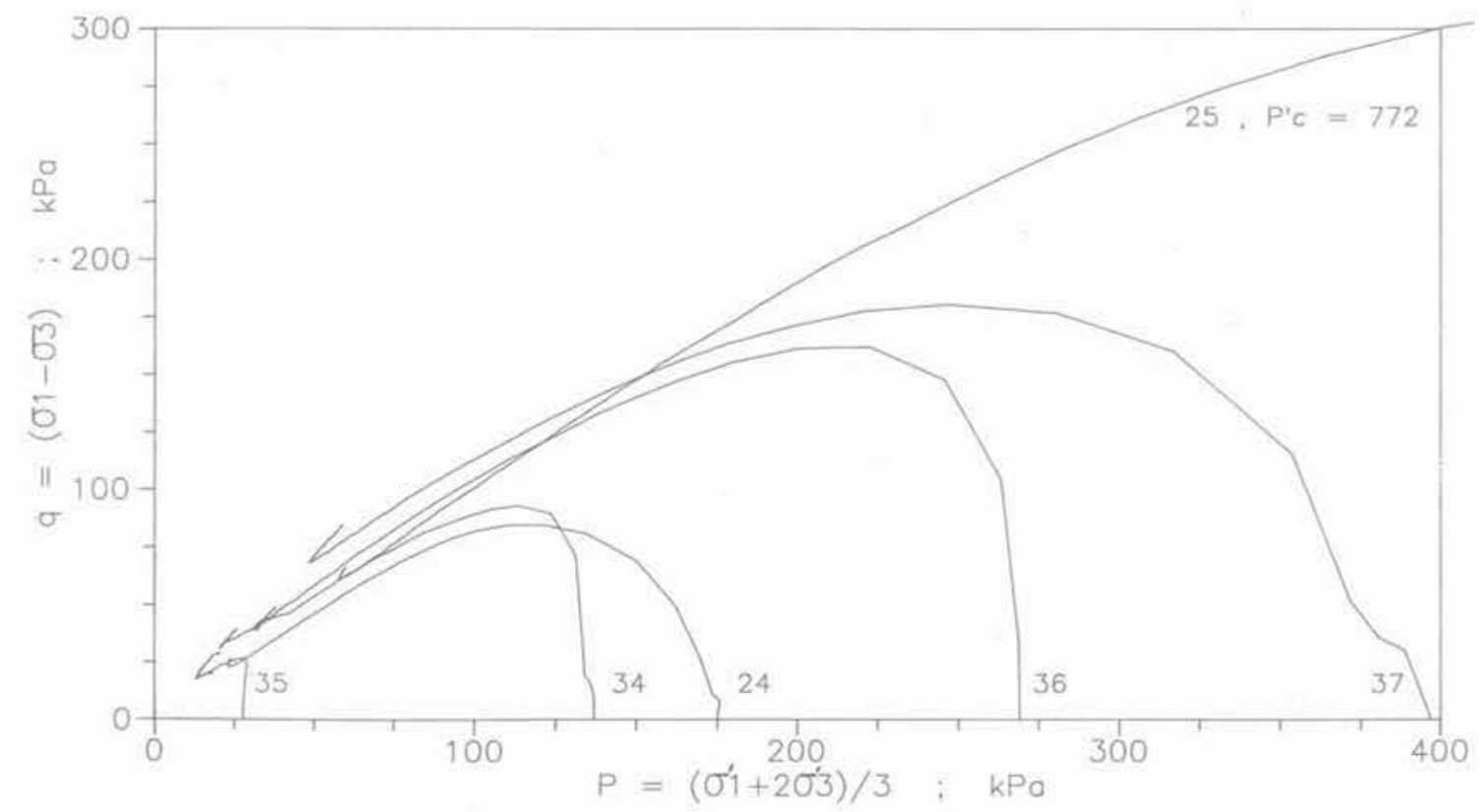

Fig. 8. - Chemins de contraintes pour les essais consolidés à un indice des vides voisin de 0,92 sous différentes pressions de consolidation.

Fig. 8. - Stress paths for tests consolidated at a voids ratio around 0.92 under different consolidation pressures. 


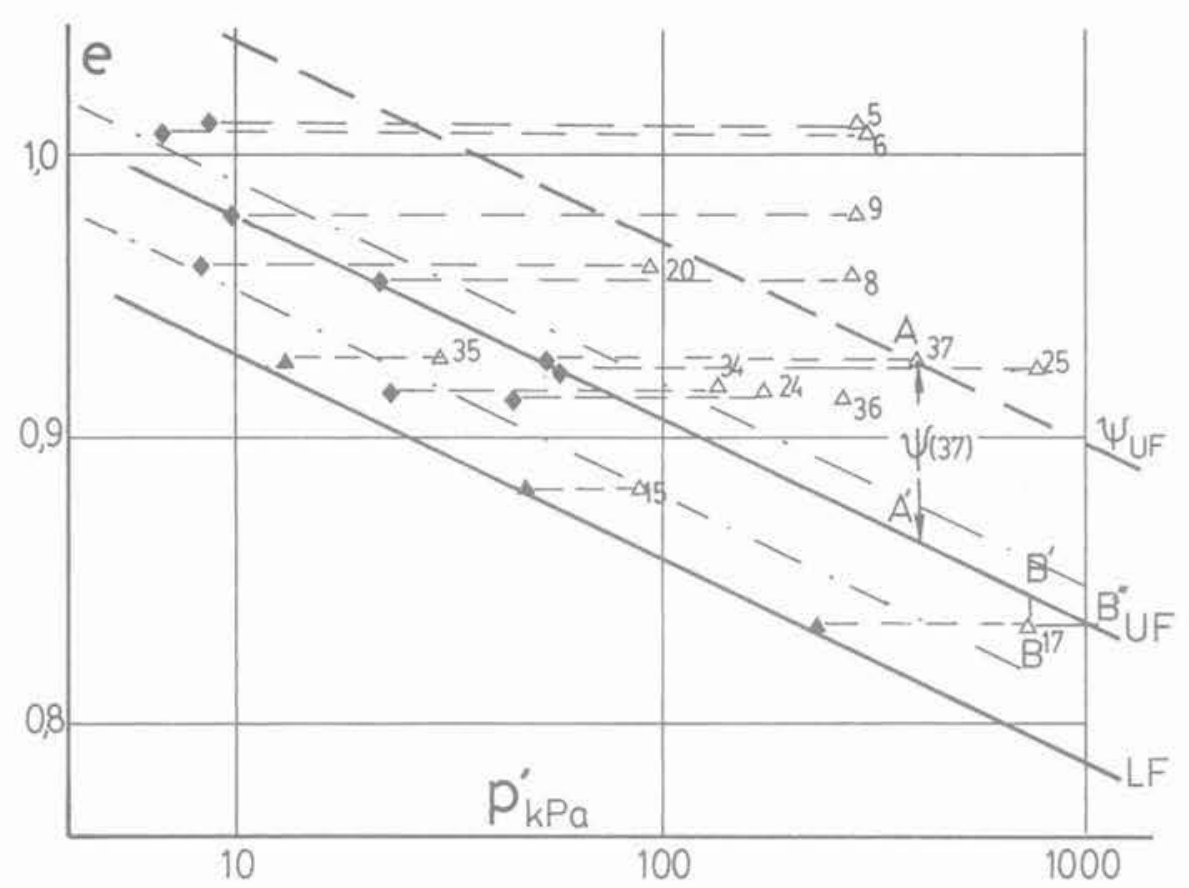

Fig. 9. - Interprétation des essais dans le plan indice des vides-pression moyenne effective des différents essais. Fig. 9. - Interpretation of the varioux tests

in a diagram voids ratio vs mean effective pressure.

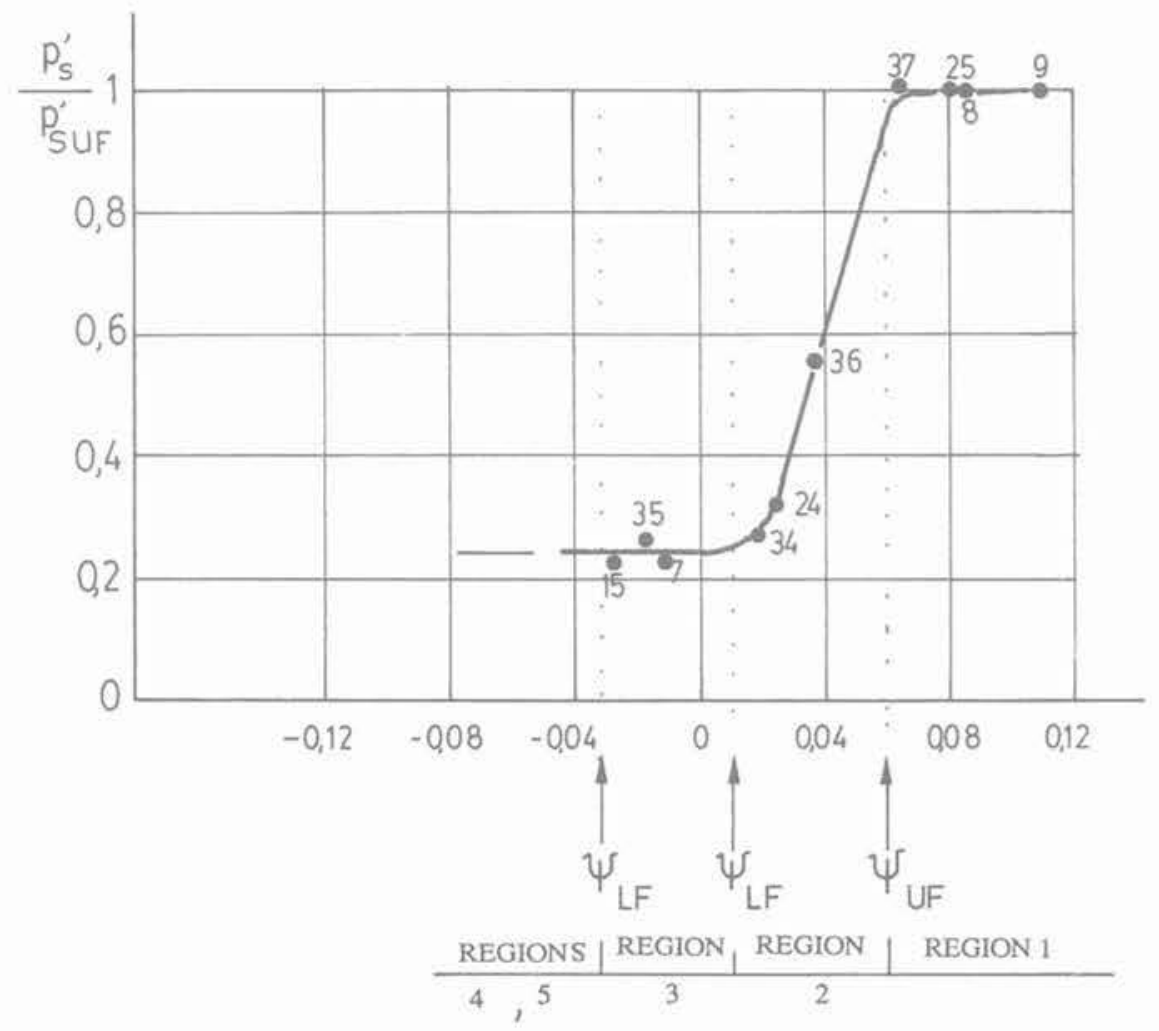

Fig. 10. - Rapport de contraintes finales en fonction du paramètre $\Psi_{U F}$. Fig. 10. - Ratio of the final mean effective pressure vs state parameter $\Psi_{U F}$ 
de plasticité parfaite. Par exemple, pour l'essai 37. $\mathrm{p}_{\text {sUF }}$ est donné par l'abscisse de l'intersection d'une droite horizontale avec une ordonnée égale à 0,928 et de la courbe UF, soit $50 \mathrm{kPa}$. Le rapport p's/p'suF est donc égal à 1,02 puisqu'à l'état de plasticité parfaite la contrainte effective moyenne était égale à 57 $\mathrm{kPa}$. La valeur du paramètre d'état est donnée par la distance verticale entre les points $A$ et $A^{\prime}$ sur la figue 10 , soit 0,062 . Pour l'essai $17, p_{\text {suF }}$ est donné par l'abscisse du point B", soit $1005 \mathrm{kPa}$, et le rapport $\mathrm{p}_{\mathrm{s}}^{\prime} / \mathrm{p}_{\mathrm{suF}}^{\prime}$ vaut par conséquent 0,228 . Le paramètre d'état après consolidation est donné par le vecteur BB', soit - 0,012.

La figure 10 indique que lorsque le paramètre d'état est supérieur à $+0,06$, l'état de plasticité parfaite obtenu lors d'essai de compression dans la cellule triaxiale correspond à des conditions données par la courbe UF. La courbe $\Psi$ (UF) est donc la limite inférieure de la région 1 proposée par KONRAD (1990). Pour le sable d'Hostun RF, $\Psi$ (UF) est égal à $+0,06$. Pour des valeurs de $\Psi$ i inférieures à $\Psi(\mathrm{UF})$ et supérieures à $\Psi\left(\mathrm{LF}_{+}\right)$, le rapport p's/p'sUF décroît quasi linéairement jusqu'à une valeur égale à 0,25 pour $\Psi \mathrm{i}$ $=\Psi(\mathrm{LF}+)=+0,01$. Finalement, lorsque le paramètre d'état est tel que $\Psi(\mathrm{LF}-)<\Psi \mathrm{i}<\Psi(\mathrm{LF}+)$, le rapport p's/p'suF est constant et vaut environ 0,24 . Ce rapport correspond en fait à la valeur de $\mathrm{p}_{\text {sLF }}^{\prime} / \mathrm{p}_{\mathrm{sUF}}^{\prime}$, et quantifie la différence entre les contraintes effectives moyennes maximum et minimum à l'état de plasticité parfaite pour le sable d'Hostun.

\section{CONCLUSIONS}

Les conclusions suivantes peuvent être tirées des résultats des essais de compression non drainés en cellule triaxiale sur des échantillons saturés de sable d'Hostun lâche.

1. Le comportement non drainé du sable d'Hostun dépend à la fois de l'indice des vides et du niveau de contrainte isotrope initial, c'est-à-dire du paramètre d'état initial.

2. Les essais ont mis en évidence l'existence d'une limite supérieure (courbe UF) et inférieure (courbe LF) des contraintes effectives moyennes obtenues à l'état de plasticité parfaite pour le même sable. Les conditions à l'état de plasticité parfaite sont définies uniquement en fonction de la valeur du paramètre d'état initial.

3. Pour une valeur du paramètre d'état supérieure à $+0,06$, les contraintes effectives moyennes à l'état de plasticité parfaite seront données par la courbe UF. Par contre, si le paramètre d'état initial est entre - 0,03 et + 0,01, l'état de plasticité parfaite sera caractérisé par la courbe LF. Pour un paramètre d'état intermédiaire, c'est-à-dire entre $+0,01$ et $+0,06$, les contraintes effectives moyennes à l'état de plasticité parfaite seront intermédiaires entre celles données par les courbes UF et LF. II apparaît que la relation entre ces contraintes et le paramètre d'état est linéaire.

Pour un sable à un indice des vides donné, les conditions caractérisant l'état de plasticité parfaite ne sont donc pas uniques.
4. Les résultats de ces essais sont conformes aux travaux récents de KONRAD (1990) sur des sables ayant des caractéristiques physiques bien différentes de celles du sable d'Hostun. L'étude effectuée a permis d'étendre les concepts proposés par KONRAD (1990) pour le comportement non drainé des sables lâches au sable d'Hostun RF.

\section{REMERCIEMENTS}

Les auteurs remercient le Centre Jacques-Cartier et son Directeur Alain Bideau pour l'aide apportée à la réalisation de cette étude, ainsi que le Greco \& Rhéologie des Géomatériaux ».

\section{BIBLIOGRAPHIE}

ALARCON-GUZMAN A., LEONARD G.A., CHAMEAU J.L. (1988), Undrained monotonic and cyclic strenght of sands. Journal of the geotechnical Engineering Division. Proc of the ASCE. Vol. $114 \mathrm{n}^{\circ} 10$, October 1988, pp. 1089-1109.

BEEN K., JEFFERIES M.G. (1985), A state parameter for sands, Geotechnique Vol. $35 n^{\circ} 2$, June 1985, pp. 99-112.

BIAREZ J., GRESILLON J.M. (1972), Essais et sug. gestion pour le calcul de la force portante des pieux en milieu pulvérulent. Géotechnique, Vol. $22, n^{\circ} 2$, pp. 433-450.

BOUSQUET H. (1988), Etude en laboratoire du comportement non drainé d'un sable lâche et application à la liquéfaction statique, rapport de DEA, Institut de Mécanique de Grenoble, Sept. 1988.

CANOU J. (1989), Contribution à létude et à l'élaboration des propriétés de liquéfaction d'un sable. Thèse de Doctorat, préparée au CERMES-ENPC. soutenue le 20.03.89.

CASAGRANDE A. (1936), Characteristics of cohesionless soils affecting the stability of earth fills. Journal of the Boston Society of Civils Engineer. Janvier 1936.

CASTRO G. (1969), Liquefaction of sands. Thesis, Harvard Soil Mechanics Series n81. Harvard University Cambridge Massachussetts.

COLLIAT J.L., DESRUES J., FLAVIGNY E. (1986), Avantages et inconvénients de l'utilisation d'un système d'antifrettage dans l'essai triaxial de révo. lution. Revue Française de Géotechnique n ${ }^{\circ} 34$, pp. 41-55.

DJEDID A. (1986), Etude du comportement non drainé du sable. Mémoire de DEA, Institut de Mécanique de Grenoble. Sept. 1986.

FLAVIGNY E., DESRUES J., PALAYER B. (1990), Le sable d'Hostun RF. Note technique, Revue Française de Géotechnique n 53, pp. 67-69.

FLAVIGNY E., FORAY P, DARVE F. (1979), Undrainded test of sand. Discussion, Journal of the Geotechnical Engineering Division. Proc. of the ASCE, Vol. 105, n० 9, sept. 1979. pp. 1126-1129.

FORAY P., PUECH A. (1976), Influence de la com. pressibilité sur la force portante à la rupture des 
pieux en milieux pulvérulent. Annales ITBTP, Série Sols et Fondations, $n^{\circ}$ 141, Mai 1976, $n^{\circ} 339$.

KONRAD J.M. (1990), The minimum undrained strength of two sands. Accepted for publication. Journal of the Geotechnical Engineering Division. Proc, of the ASCE, n GT6 (4), pp. 932-947.

KRAMER S.L., SEED B. (1988), Initiation of soil liquefaction under static loading conditions. Journal of the geotechnical Engineering Division. Proc. of the ASCE, Vol. 114, $n^{\circ} 4$, Avril 1988, pp. $412-430$

SHOLSSER F., CORTÉ J.-F., DORMIEUX L. (1987), Effets dynamiques de leau dans les sols. Rapport général, 9e Congrès Européen de Mécanique des sols et travaux de Fondation, Dublin, pp. 6.1-6.49.

SLADEN J.A., D'HOLLANDER R.D., KRAHN J. (1985), The liquefaction of sands, a collapse surface approach, Canad. Geotechnical journal, Vol. 22, n 4 , Dec. 1985 , pp. 564-579. 